\title{
Lidil
}

Revue de linguistique et de didactique des langues

$52 \mid 2015$

Les pratiques artistiques dans l'apprentissage des langues

\section{Anne Godard (dir.), La littérature dans l'enseignement du FLE}

Paris, Didier, coll. « Langue et didactique », 2015

\section{Camille Vorger}

\section{OpenEdition \\ Journals}

Édition électronique

URL : http://journals.openedition.org/lidil/3900

DOI : $10.4000 /$ lidil.3900

ISSN : $1960-6052$

\section{Éditeur}

UGA Éditions/Université Grenoble Alpes

\section{Édition imprimée}

Date de publication : 20 novembre 2015

Pagination : 201-203

ISBN : 978-2-84310-312-4

ISSN : $1146-6480$

\section{Référence électronique}

Camille Vorger, « Anne Godard (dir.), La littérature dans l'enseignement du FLE », Lidil [En ligne], 52 | 2015 mis en ligne le 01 janvier 2017, consulté le 29 octobre 2020. URL : http://journals.openedition.org/lidil/ 3900 ; DOI : https://doi.org/10.4000/lidil.3900 
tion aborde également la question de l'enseignement des propositions relatives en s'appuyant sur la théorie de la transposition didactique. À partir d'une séance de classe de CM2, Kaheraoui, Coret et Volteau analysent la démarche de l'enseignant et les interactions entre l'enseignant et les élèves. Elles montrent un décalage important entre la complexité théorique de la question des propositions relatives et la grammaire didactique. On observe un lien entre cette contribution et celle de Trévisiol-Okamura analysant les manuels de FLE. Selon l'approche théorique adoptée en linguistique, la grammaire de référence ou la grammaire didactique va présenter les relatives différemment, ce qui mène souvent à la confusion et montre la difficulté du processus de transposition. Cet ouvrage, riche en apports théoriques et résultats des travaux empiriques, aborde de façon très claire la question des subordonnées relatives selon les perspectives différentes adoptées.

Tatiana Aleksandrova

LIDILEM, Université Grenoble Alpes

\section{Anne Godard (dir.), La littérature dans l'enseignement du FLE,} Paris, Didier, coll. «Langue et didactique», 2015.

Les 338 pages de cet ouvrage rassemblent les contributions d'une dizaine de didacticiens visant à dresser un état des lieux des enjeux de la littérature en FLE ainsi que des discours et des pratiques auxquelles elle donne lieu, dans des contextes d'enseignement divers. Les huit chapitres progressent d'une approche historico-théorique de la question (chapitre 1) à un état des lieux de la formation littéraire (chapitre 2), de l'enseignement de la littérature française et francophone à l'étranger (chapitre 3), des discours et pratiques institutionnels - et des ressources associées (chapitre 4) - , avant d'en arriver à une analyse approfondie de démarches créatives et interactives expérimentées autour de la littérature en FLES (chapitres 5 à 7).

Si la première partie répond à une problématique assez générale et à des questions toutes rhétoriques - «La littérature est-elle réservée à certains publics? À certains niveaux? Peut-elle être pensée d'emblée dans une progression linguistique ou sa spécificité en classe se situet-elle dans un à-côté récréatif? Comment peut-elle être didactisée tout en ménageant le plaisir comme moyen d'apprentissage ?» (p. 6) - , la 
suite a le mérite d'essayer de construire des ponts entre le communicatif et le culturel, entre les didactiques du FLM, du FLE et du FLS. De même, si le panorama des pratiques institutionnelles à l'étranger s'avère laborieux, celui des ressources nous permet d'entrer dans le vif du sujet - dans le vif de la littérature. Un tel état des lieux attire l'attention sur la difficulté d'intégrer, dans les manuels, une véritable progression autour de la littérature. Les démarches décrites en fin d'ouvrage montrent comment la perspective actionnelle permet, avec la pédagogie de projet, le décloisonnement et l'établissement d'une relation active (créative) à la langue. Enfin, l'intérêt du dernier chapitre - évoquant l'expérience de «l'étrangéité» de la langue française à travers la découverte d'auteurs québécois et d'écrivains francophones plurilingues mérite d'être souligné en vue d'une «ouverture au plurilinguisme par la littérature».

Nous ne reviendrons pas sur l'état des lieux préliminaire, si ce n'est pour souligner quelques apports originaux et transversaux : la notion allemande de «Bildung» correspond ainsi à ce que l'on nomme en France «culture humaniste», mettant l'accent sur «le développement personnel des potentialités intellectuelles, affectives et éthiques de l'être humain au sein de la collectivité dont il fait partie» (p. 64). En d'autres termes, il s'agit d'insister sur les résonances identitaires de la littérature, «comme rencontre avec soi autant qu'avec l'autre» (p. 69) et sur la capacité d'empathie comme «essence de l'esprit démocratique» (p. 71). Le panorama des dispositifs à l'étranger montre que se dessine une nouvelle géographie de la littérature, tant dans les corpus - marqués par l'abandon des hiérarchies entre littérature d'élite et littérature grand public et l'extension aux littératures contemporaines et francophones que dans les pratiques qui s'ouvrent à l'intermédialité autant qu'à la multimodalité. En outre, l'apport de la didactique du FLS est mis en avant en tant que «l'accès à la littérature est aussi un mode d'accès à la littératie» (p. 138). Le chapitre 4 s'intéresse aux collections de «lecture en français facile» où «le texte original n'est souvent qu'un palimpseste» (p. 155). Certaines d'entre elles - plus récentes, telle «Mondes en $V F »-$ offrent l'intérêt de présenter des textes originaux accessibles dès le niveau A2 et assortis d'un accompagnement didactique en ligne, démarche qui sera explicitée concernant les nouvelles de Vassilis Alexakis (p. 174). Les auteurs de l'ouvrage remarquent cependant que la toile reste un espace à investir dans ce domaine (p. 165). Le chapitre 5 s'intéresse aux écritures créatives en FLE, soulevant la question de l'évaluation et mentionnant «le paradoxe de l'écriture littéraire en 
contexte scolaire» (p. 189), alors même que l'écriture créative tend à «redonner sa place au sujet caché - et parfois nié - dans tout apprenant-écrivant» (p. 190). L'intervention d'écrivains en classe de FLE sous la forme d'ateliers d'écriture permet de désacraliser et d'ouvrir un espace de liberté dans le rapport à la langue, à l'écriture. Le recours à des carnets - avec l'exemple du «carnet d'anecdotes» (p. 198) va dans le sens d'une conscientisation des démarches d'écritures de l'apprenant. La littérature peut alors être approchée «en actes» (chapitre 6), en privilégiant le «voir, entendre, ressentir» à travers des approches multimodales. L'oralité se conjugue avec «l'auralité»-du latin os, auris, l'oreille, néologisme servant à qualifier l'attitude et le travail d'écoute active (p. 237) - grâce à différentes modalités de lectures orale, chorale, en relais, etc. Une approche originale de la poésie sonore en FLE propose de partir d'un poème de Ghérasim Luca pour se confronter au «bégaiement dans la langue» qui le caractérise (p. 240), gagner en fluidité prosodique et trouver sa voix en français. Le dernier chapitre ouvre au plurilinguisme et énumère un bel inventaire de titres possibles autour de cette question, citant des auteur.e.s aux parcours édifiants (p. 286-287) tels Nancy Huston, Akira Mizubayashi ou encore Agota Kristof, à laquelle nous laisserons le mot de la fin (citée p. 295) :

Je sais lire, je sais de nouveau lire. Je peux lire Victor Hugo, Rousseau, Voltaire, Sartre, Camus, Michaux, Francis Ponge, Sade, tout ce que je veux lire en français.

Camille Vorger

Université de Lausanne (EFLE)

Steven McCafferty et Gale Stam (eds), Gesture. Second Language Acquisition and Classroom Research, Londres, Routledge, 2008, $327 \mathrm{p}$.

Le présent recueil est l'un des rares ouvrages qui aient été entièrement dédiés, à présent, aux fonctions des gestes dans l'acquisition d'une langue seconde, y compris dans le contexte de la classe de langue. Peu connu en France, il a le mérite de faire le point sur une nouvelle piste de recherche au sein des études actuelles sur la gestualité, domaine de recherche qui est en pleine croissance depuis les années quatre-vingt, 